United Nations Educational Scientific and Cultural Organization

and

International Atomic Energy Agency

THE ABDUS SALAM INTERNATIONAL CENTRE FOR THEORETICAL PHYSICS

\title{
MAGNETIZATION REVERSAL THROUGH SOLITON FLIP IN A BIQUADRATIC FERROMAGNET WITH VARYING EXCHANGE INTERACTIONS
}

\author{
M. Daniel ${ }^{1}$ \\ Centre for Nonlinear Dynamics, Department of Physics, \\ Bharathidasan University, Tiruchirapalli - 620 024, India \\ and \\ The Abdus Salam International Centre for Theoretical Physics, Trieste, Italy \\ and \\ L. Kavitha ${ }^{2}$ \\ Department of Physics, Lady Doak College, Madurai - 625 002, India \\ and \\ The Abdus Salam International Centre for Theoretical Physics, Trieste, Italy.
}

\begin{abstract}
We study the phenomenon of magnetization reversal in the form of a soliton flip in a biquadratic ferromagnetic spin chain induced by varying bilinear and biquadratic exchange interactions. This is carried out by analysing the evolution of the velocity and amplitude of the soliton using a perturbation analysis.
\end{abstract}

\section{MIRAMARE - TRIESTE}

July 2002

\footnotetext{
${ }^{1}$ Regular Associate of the Abdus Salam ICTP. Corresponding author: muthiahdaniel@yahoo.co.uk

2 kavithalouis@yahoo.com
} 


\section{Introduction}

Magnetization reversal process or switching properties through understanding of the underlying magnetization dynamics in magnetic systems is an important issue mainly because the dynamic process is nonlinear in nature. The importance of the issue is also based on the fact that success and development of random access memories depend on the magnetization switching process. Magnetization reversal process is normally based on coherent rotation of magnetization and propogation of domain walls in the presence of the magnetic field and this has been studied in detail[1,2]. One cannot rule out the possibility of magnetization reversal without applying external magnetic fields. Among various approaches, magnetization switching by stress induced anisotropy and thermal activation assume importance[3,4]. In our present study, we propose that site-dependent or inhomogeneous exchange interactions can also be a good candidate for activating magnetization or spin reversal process in a ferromagnet. Most of the available results on magnetization reversal process is based on experimental studies and numerical simulations and analytical results are very limited. Recently, it was found that the classical Landau-Lifshitz(LL) equation is an useful model to describe fast magnetization reversal process[5,6]. In entirely different context, the LL equation[7], corresponding to different magnetic interactions has been proved to be completely integrable admiting soliton solutions in several cases[8-10]. Thus soliton has been identified as very useful object that can describe localized coherent spin or magnetization configurations in classical ferromagnetic systems. Higher order magnetic interactions, inhomogeneous external magnetic fields etc. introduce perturbation to these solitons $[9,10]$. Thus it has become increasingly important to investigate the effect of these perturbation on the soliton and in particular a study to understand whether any of these perturbations can contribute to magnetization reversal or spin flip in ferromagnetic system, needs immediate attention. In this paper, we try to find answer for the question whether varying (inhomogeneous) exchange interactions can induce magnetization reversal process in a biquadratic ferromagnetic system.

\section{Dynamics of biquadratic ferromagnet with varying exchange interactions}

The Hamiltonian for a classical Heisenberg ferromagnetic spin chain with varying bilinear and biquadratic exchange interactions can be written as

$$
\mathcal{H}=-\sum_{i}\left[J_{e} f_{i}\left(\mathbf{S}_{i} . \mathbf{S}_{i+1}\right)+J_{b} g_{i}\left(\mathbf{S}_{i} . \mathbf{S}_{i+1}\right)^{2}\right], \mathbf{S}_{i}=\left(S_{i}^{x}, S_{i}^{y}, S_{i}^{z}\right),
$$

where $J_{e}$ and $J_{b}$ are the bilinear and biquadratic exchange parameters respectively and $f_{i}$ and $g_{i}$ characterize the variation of the bilinear and biquadratic exchange interactions at the lattice sites

along the spin chain. The Landau-Lifshitz(LL) equation of motion[7] corresponding to the spin Hamiltonian (1) in the case of a one dimensional classical continuum spin chain can be obtained 
from the lattice equation $\frac{d \mathbf{S}_{i}}{d t}=\left\{\mathbf{S}_{i}, \mathcal{H}\right\}_{P B}$, and by taking the continuum limit by expanding $\mathbf{S}_{n \pm 1}$ and $f_{n-1}, g_{n-1}$ about $\mathbf{S}(x, t)$ and $f(x), g(x)$ in Taylor expansions where $\mathrm{x}$ is a continuous variable[11]. The resultant LL equation of motion reads

$$
\begin{aligned}
\mathbf{S}_{t}= & \mathbf{S} \wedge\left\{\left[A-\frac{a}{2} A_{x}+\frac{a^{2}}{2} A_{x x}+\frac{a^{2}}{2} J_{b} g\left(\mathbf{S} . \mathbf{S}_{x x}\right)\right] \mathbf{S}_{x x}+\left[A_{x}-\frac{a}{2} A_{x x}\right.\right. \\
& \left.\left.+\frac{a^{2}}{3} J_{b} g\left(\mathbf{S} . \mathbf{S}_{x x x}\right)\right] \mathbf{S}_{x}+\frac{a^{2}}{12} A \mathbf{S}_{x x x x}+\frac{a^{2}}{6} A_{x} \mathbf{S}_{x x x}\right\}, \quad \mathbf{S}^{2}=1,
\end{aligned}
$$

where $A(x)=J_{e} f(x)+J_{b} g(x)$. Here, the suffices $\mathrm{t}$ and $\mathrm{x}$ represent partial derivatives with respect to $t$ and $x$ respectively. As the LL equation (2) is a nontrivial vector nonlinear partial differential equation, it is difficult to solve it in its natural vector form to understand the underlying nonlinear spin dynamics. Hence, we intend to map this to one of the well known nonlinear evolution equations that admits solitons. For this, we map the above spin chain at a given instant of time onto a moving helical space curve in $E^{3}$ which is described by the motion of a local coordinate system(orthogonal trihedral) on the curve. The change in orientation of the orthogonal trihedral $\mathbf{e}_{i}, i=1,2,3$ which defines the space curve within rigid motions is determined by the Serret-Frenet(S-F) equations $\mathbf{e}_{i x}=\mathbf{D} \wedge \mathbf{e}_{i}$ where $\mathbf{D}=\kappa \mathbf{e}_{3}+\tau \mathbf{e}_{1}$ and $\kappa \equiv\left(\mathbf{e}_{1 x} \cdot \mathbf{e}_{1 x}\right)^{\frac{1}{2}}$ and $\tau \equiv \frac{1}{\kappa^{2}} \mathbf{e}_{1} \cdot\left(\mathbf{e}_{1 x} \wedge \mathbf{e}_{1 x x}\right)$ are the curvature and torsion of the space curve[12] respectively which are related to the energy and current densities of the spin system. In view of the above identification the equation of motion (2) can be rewritten by replacing $\mathbf{S}(x, t)$ by $\mathbf{e}_{1}(x, t)$ and then using the $\mathrm{S}-\mathrm{F}$ equations, the evolution equations for the trihedral $\mathbf{e}_{i}, i=1,2,3,\left(\mathbf{e}_{i t}\right)$ can be constructed. The compatibility of the trihedral namely $\left(\mathbf{e}_{i x}\right)_{t}=\left(\mathbf{e}_{i t}\right)_{x}$ leads to a set of highly nonlinear coupled evolution equations for the curvature and torsion of the space curve which on using the transformation[8], $q=\frac{\kappa}{2} \exp i \int_{-\infty}^{x} \tau\left(x^{\prime}, t\right) d x^{\prime}$, reduce to the following inhomogeneous generalized higher order nonlinear Schrödinger (NLS) equation.

$$
\begin{aligned}
& i q_{t}+(k q)_{x x}+2 k|q|^{2} q+2 q \int_{-\infty}^{x} k_{x^{\prime}}|q|^{2} d x^{\prime}+\frac{a^{2} A}{12}\left[q_{x x x x}+K_{1}|q|^{2} q_{x x}\right. \\
& \left.+K_{2} q^{2} q_{x x}^{*}+K_{3}\left|q_{x}\right|^{2} q+K_{4} q^{*} q_{x}^{2}+3 K_{2}|q|^{4} q\right]+\frac{a^{2}}{12} A_{x}\left[q_{x x x}+6|q|^{2} q_{x}\right]=0
\end{aligned}
$$

where $k(x)=\tilde{A}+\frac{a^{2}}{6} A_{x x}, K_{1}=-12\left(1+\frac{4 J_{b} g}{A}\right), \quad K_{2}=-8\left(1+\frac{3 J_{b} g}{A}\right), \quad K_{3}=-36\left(1+\frac{8 J_{b} g}{3 A}\right), \quad K_{4}=$ $-14\left(1+\frac{24 J_{b} g}{7 A}\right)$. Thus, Eq.(3) describes the dynamics of spins in an inhomogeneous classical continuum biquadratic Heisenberg ferromagnetic spin chain in an equivalent representation. A Painlevé singularity structure analysis[13] brings out the completely integrable models underlying Eq.(3) at different orders and the integrability properties were studied in detail[8,9,14-16]. Therefore, we desist from presenting more results about this aspect here. The constraint on the inhomogeneity in the form of a linear function of $\mathrm{x}$ for integrability rises an important question that what would be the effect of noninear inhomogeneity on the soliton which forms the major concern of the rest of the paper. 
Effect of nonlinear inhomogeneity on the spin soliton and magnetization reversal

We consider the inhomogeneous nonlinear equation from Eq.(3) at the order $O\left(a^{0}\right)$ and put $k(x)=k_{0}+\lambda k_{1}(x)$ where $\lambda$ is a small parameter and $k_{1}(x)$ is a nonlinear function of $\mathrm{x}$. After suitable rescaling and redefinition of $\lambda$, the equation reads

$$
i q_{t}+q_{x x}+2|q|^{2} q+\lambda\left[\left(k_{1} q\right)_{x x}+2 k_{1}|q|^{2} q+2 q \int_{-\infty}^{x} k_{1 x^{\prime}}|q|^{2} d x^{\prime}\right]=0 .
$$

We study the effect of nonlinear inhomogeneity on the spin soliton by treating terms proportional to $\lambda$ in Eq.(4) as a weak perturbation using multiple scale perturbation analysis[17]. It amy be noted that while writing Eq.(4), we have dropped terms at $O\left(a^{2}\right)$ because inhomogeneity does not enter at that order. Also, in our earlier studies we have shown that these terms at $O\left(a^{2}\right)$ as a perturbation do not alter the velocity and amplitude of the unperturbed soliton[9]. When $\lambda=0$, Eq.(4) reduces to the completely integrable cubic NLS equation which admits envelope one soliton in the form $q=\eta \operatorname{sech} \eta\left(\theta-\theta_{0}\right) \exp \left[i \xi\left(\theta-\theta_{0}\right)+i\left(\sigma-\sigma_{0}\right)\right]$, where $\theta_{t}=-2 \xi, \theta_{x}=$ $1, \sigma_{t}=\eta^{2}+\xi^{2}, \sigma_{x}=0$. Writing $\eta, \xi, \theta, \theta_{0}$ and $\sigma_{0}$ as functions of a new time scale $T=\lambda t$, and $q=\hat{q}(\theta, T ; \lambda) \exp \left[i \xi\left(\theta-\theta_{0}\right)+i\left(\sigma-\sigma_{0}\right)\right]$, under the assumption of quasi-stationarity, on expanding $\hat{q}$ in terms of $\lambda$ as $\hat{q}(\theta, T ; \lambda)=\hat{q}_{0}(\theta, T)+\lambda \hat{q}_{1}(\theta, T)+\ldots$ where $\hat{q}_{0}=\eta \operatorname{sech} \eta\left(\theta-\theta_{0}\right)$, at $O(\lambda)$ after substituting $\hat{q}_{1}=\left(\phi_{1}+i \psi_{1}\right),\left(\phi_{1}, \psi_{1}\right.$ : real $)$ we obtain

$$
\begin{aligned}
& \mathcal{L}_{1} \phi_{1} \equiv-\eta^{2} \phi_{1}+\phi_{1 \theta \theta}+6 \hat{q}_{0}^{2} \phi_{1}=F_{1}\left(\hat{q}_{0}\right), \\
& \mathcal{L}_{2} \psi_{1} \equiv-\eta^{2} \psi_{1}+\psi_{1 \theta \theta}+2 \hat{q}_{0}^{2} \psi_{1}=F_{2}\left(\hat{q}_{0}\right),
\end{aligned}
$$

where $F_{1}\left(\hat{q}_{0}\right)$ and $F_{2}\left(\hat{q}_{0}\right)$ are given by $F_{1}\left(\hat{q}_{0}\right)=-\left[\xi_{T}\left(\theta-\theta_{0}\right)-\xi \theta_{0 T}\right]+\left(k_{1} \hat{q}_{0}\right)_{\theta \theta}$ $-k_{1} \xi^{2} \hat{q}_{0}+2 k_{1}\left|\hat{q}_{0}\right|^{2} \hat{q}_{0}+2 \hat{q}_{0} \int_{-\infty}^{\theta} k_{1 \theta^{\prime}}\left|\hat{q}_{0}\right|^{2} d \theta^{\prime}$ and $F_{2}\left(\hat{q}_{0}\right)=\left[-\hat{q}_{0 T}+2\left(h_{1} \hat{q}_{0}\right)_{\theta} \xi\right] . \mathcal{L}_{1}$ and $\mathcal{L}_{2}$ are self-adjoint operators. As $\hat{q}_{0 \theta}$ and $\hat{q}_{0}$ are solutions of the homogeneous parts of Eqs.(5a) and (5b) respectively, the secularity conditions give

$$
\int_{-\infty}^{\infty} \hat{q}_{0 \theta} F_{1} d \theta=0, \quad \int_{-\infty}^{\infty} \hat{q}_{0} F_{2} d \theta=0
$$

The effect of inhomogeneity in exchange interactions " $k_{1}$ " on spin soliton $\hat{q}_{0}$ can be understood by evaluating the above two integrals for specific forms of " $k_{1}$ ". As it is known that the model supports soliton spin excitations when the inhomogeneity is in the form of a linear function of $\mathrm{x}[14,15]$, in this paper, we consider (i) a quadratic-type inhomogeneity represented by $k_{1}(x)=\beta_{2} x^{2}+\beta_{1} x+\beta_{0}$ and (ii) a more complicated localized inhomogeneous exchange interaction represented in terms of $k_{1}(x)=\beta_{3} \tanh \eta_{p} x$ where $\beta_{0}, \beta_{1}, \beta_{2}$, and $\beta_{3}$ are constants and $\eta_{p}$ replaces the $\eta$ in the solution.

\section{A. Quadratic inhomogeneity}

Substituting the quadratic form of inhomogeneity in the secularity conditions (6), we obtain

$$
\xi_{s T}=2 \hat{\beta}\left(\eta_{s}^{2}-\xi_{s}^{2}\right), \quad \eta_{s T}=2 \hat{\beta} \xi_{s}\left(\eta_{s}-2\right),
$$




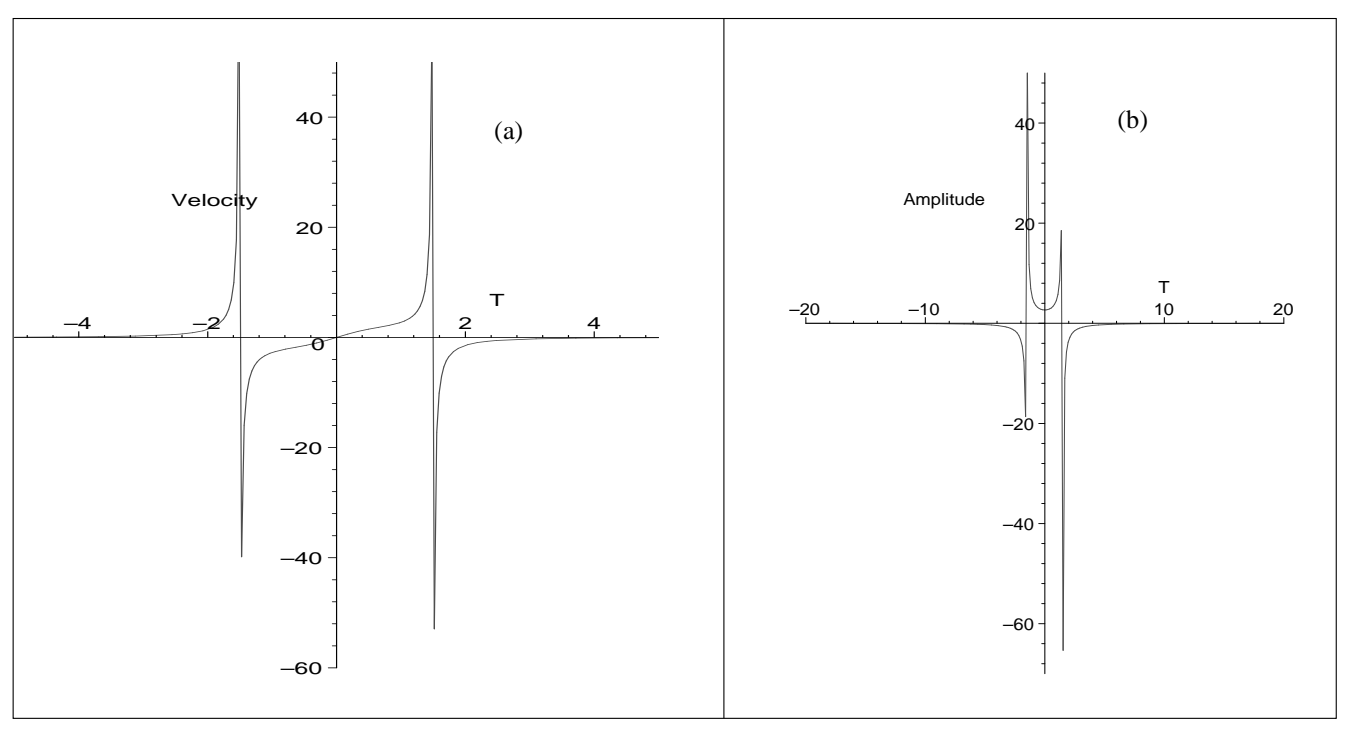

FIG. 1: (a) Time evolution of the velocity $\left(\xi_{s}\right)$ of the soliton and (b) soliton flip in terms of amplitude $\left(\eta_{s}\right)$, when $C_{s 0}=0$ under quadratic inhomogeneity with the initial amplitude $\eta_{s 0}=3.0$.

where $\xi_{s}$ and $\eta_{s}$ are the velocity and amplitude of the soliton in this case (instead of $\eta$ and $\xi$ ), $\hat{\beta}=\left(\beta_{1}+\beta_{4}\right)$ and $\beta_{4}=\beta_{2} \eta_{s}\left[\left(\theta-\theta_{0}\right) \tanh \eta_{s}\left(\theta-\theta_{0}\right)\right]_{-\infty}^{\infty}$ which is assumed to be finite by choosing $\theta_{0}$ appropriately. When the inhomogeneity is absent, the velocity and amplitude of the soliton remain constant which is evident from Eq.(7). To understand the nature of evolution of the amplitude and velocity of the soliton under the quadratic form of inhomogeneity, we solve the set of coupled equations (7). We differentiate the second of Eq.(7) and use the first one in the resultant equation to get after integrating once (and suitable rescaling of T) $\eta_{s T}^{2}-\frac{1}{2} \eta_{s}^{4}+\frac{4}{3} \eta_{s}^{3}=C_{s 0}$, where $C_{s 0}$ is the constant of integration. In the above equation $C_{s 0}$ is found to be equivalent to the energy of the soliton which oscillates under the potential $\left(-\frac{1}{2} \eta_{s}^{4}+\frac{4}{3} \eta_{s}^{3}\right)$.

When $C_{s 0}=0$, the amplitude and velocity of the soliton are found to be respectively $\eta_{s}=$ $-6 Q^{-1}, \quad \xi_{s}=\frac{3}{K Q(Q+3)}\left[1-3\left(1-\frac{8}{3 \eta_{0 s}}\right)^{\frac{1}{2}}-2 T\right]$, where $Q=\left[\left(T-\frac{1}{2}\right)^{2}+3\left(1-\frac{8}{3 \eta_{s 0}}\right)^{\frac{1}{2}}\left(T-\frac{1}{2}\right)-\frac{6}{\eta_{s 0}}\right]$ and $\eta_{s 0}$ is the initial amplitude of the soliton. Assuming that the soliton with an initial amplitude of $\eta_{s 0}=3.0$, starts from rest at $T=0$, we have plotted in Figs.1a and 1b, the velocity and the amplitude of the soliton. From the figures we observe that as time passes on the velocity and amplitude of the soliton increase and when reaching a maximum value suddenly flips leading to magnetization reversal and moves in the opposite direction. Then the soliton dies out slowly due to inhomogeneity of the exchange interactions along the spin chain. As the velocity of the soliton is inversely proportional to the inhomogeneity, the soliton damps very quickly in the case of highly inhomogeneous medium. The soliton would have exploded had it not flipped and reversed when it moves with high speed and fast growing amplitude. 


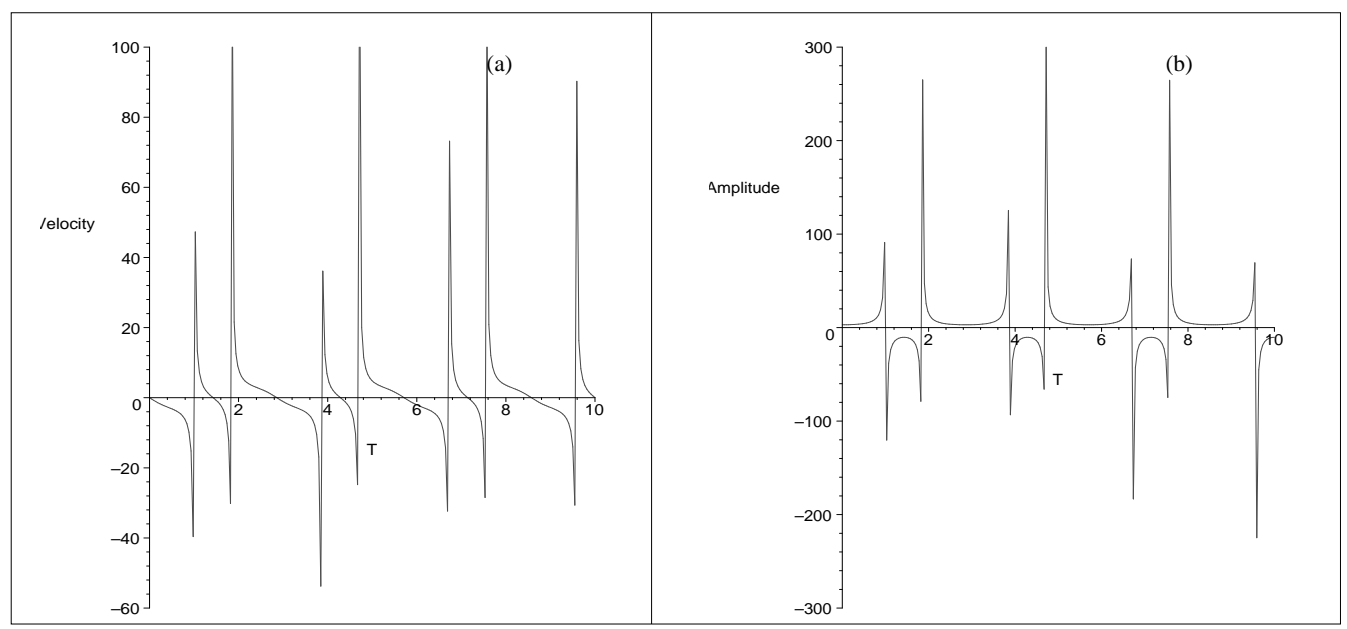

FIG. 2: (a) Time evolution of the velocity $\left(\xi_{s}\right)$ of the soliton and (b) soliton flip in terms of amplitude $\left(\eta_{s}\right)$, when $C_{s 0}=\frac{7}{81}$ under quadratic inhomogeneity with the initial amplitude $\eta_{s 0}=3.0$.

When $C_{s 0} \neq 0$, the equation can be integrated and the solution and hence the amplitude $\eta_{s}$ and velocity $\xi_{s}$ of the soliton can be expressed in terms of Jacobian elliptic functions[18]. The amplitude has the form $\eta_{s}(T)=\frac{\left(\epsilon_{-}-C_{2} \epsilon_{+}\right)-\epsilon_{+} c n\left(\frac{T}{g}\right)}{\left(\delta_{-}-C_{2} \delta_{+}\right)-\delta_{+} c n\left(\frac{T}{g}\right)}$, where $\epsilon_{ \pm}=\eta_{2} a_{2} \pm \eta_{1} b_{2}, \quad \delta_{ \pm}=a_{2} \pm b_{2}$, $C_{2}=\frac{2\left(\eta_{1}-\eta_{s 0}\right) b_{2}}{\eta_{s 0} \delta_{+}-\epsilon_{+}}, \quad g=\frac{1}{\sqrt{a_{2} b_{2}}}, \quad a_{1}^{2}=\frac{-\left(\eta_{3}-\eta_{3}^{*}\right)^{2}}{4}, \quad b_{1}=\frac{\left(\eta_{3}+\eta_{3}^{*}\right)}{2}, \quad a_{2}^{2}=\left(\eta_{1}-b_{1}\right)^{2}+a_{1}^{2}, \quad b_{2}^{2}=$ $\left(\eta_{2}-b_{1}\right)^{2}+a_{1}^{2}$. Here $\eta_{s 0}$ is the initial amplitude $\eta_{s}(0)$ of the soliton at $T=0$ and $\eta_{1}$ and $\eta_{2}$ are the two real roots of the polynomial $\eta_{s}^{4}-\frac{8}{3} \eta_{s}^{3}+\frac{7}{81}=0, \eta_{3}$ is the complex root of the same and $\eta_{3}^{*}$ its complex conjugate. The velocity $\xi_{s}$ of the soliton is of the form $\xi_{s}=$ $\frac{\left(\epsilon_{+} \delta_{-}-\delta_{+} \epsilon_{-}\right) \operatorname{sn}\left(\frac{T}{g}\right) d n\left(\frac{T}{g}\right)}{K g\left\{\left[\left(\delta_{-}-C_{2} \delta_{+}\right)-\delta_{+} c n\left(\frac{T}{g}\right)\right]\left[\left(\epsilon_{-}-C_{2} \epsilon_{+}\right)-\epsilon_{+} c n\left(\frac{T}{g}\right)\right]-2\left[\left(\delta_{-}-C_{2} \delta_{+}\right)-\delta_{+} \operatorname{cn}\left(\frac{T}{g}\right)\right]^{2}\right\}}$. Here $\operatorname{sn}\left(\frac{T}{g}\right), \operatorname{cn}\left(\frac{T}{g}\right)$ and $d n\left(\frac{T}{g}\right)$ are Jacobian elliptic functions. While evaluating the above expressions for amplitude and velocity $C_{s 0}$ is chosen as $\frac{7}{81}$ for convenience.

The above expressions for the velocity $\left(\xi_{s}\right)$ and amplitude $\left(\eta_{s}\right)$ of the soliton have been plotted in Figs.2a and 2b. The figures show that unlike the case corresponding to $C_{s 0}=0$, here the flipping of the soliton amplitude or magnetization reversal and the reversal of the velocity of the soliton occur doubly periodically and continues indefinitely. The soliton in this case does not die out. This is because as mentioned earlier, $C_{s 0}$, now acts as a source of energy for soliton flip or for magnetic reversal, sustaining the soliton to oscillate doubly periodically under the potential $\left(\frac{-\eta_{s}^{4}}{2}+\frac{4}{3} \eta_{s}^{3}\right)$ (forced oscillations) indefinitely. 


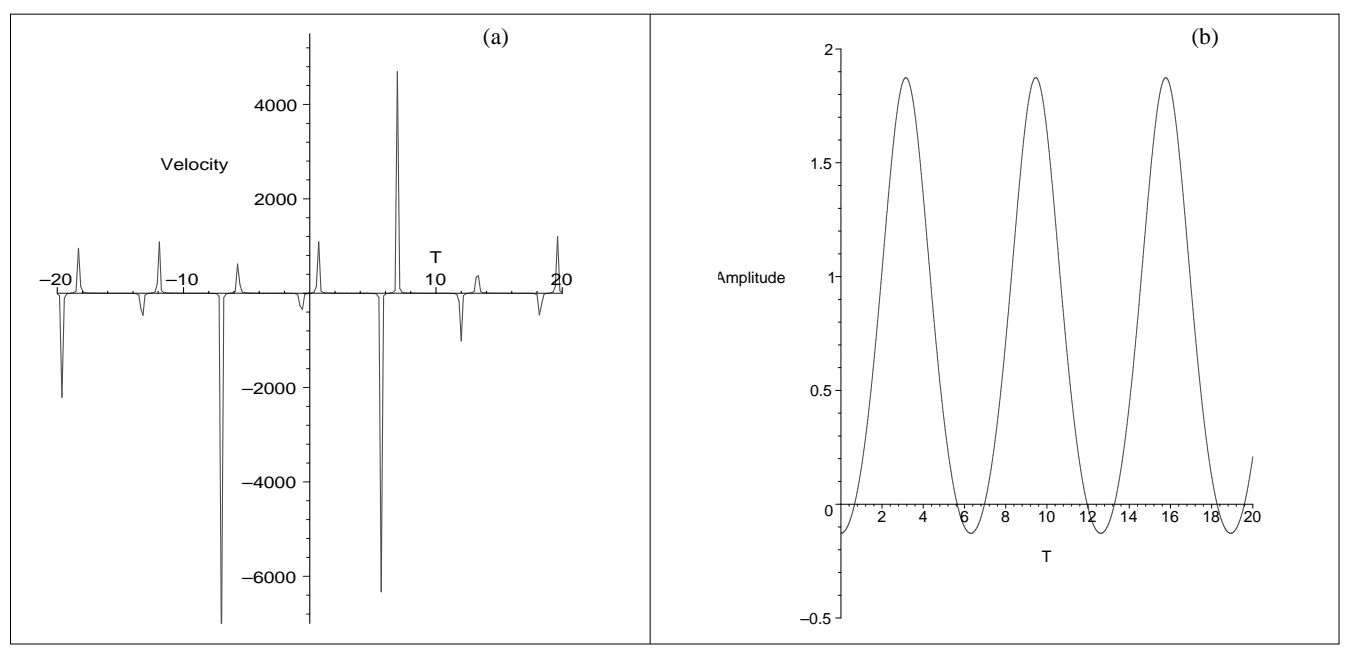

FIG. 3: Time evolution of (a) the velocity $\left(\xi_{p}\right)$ and (b) the amplitude $\left(\eta_{p}\right)$ of the soliton under tangent hyperbolic inhomogeneity when the initial amplitude is $\eta_{p 0}=\frac{1}{2 \sqrt{3}} \frac{1-\sqrt{3}}{(1+\sqrt{3}) 2}$.

\section{B. Localized inhomogeneity}

As a second example, we consider the inhomogeneity of the exchange interactions in the form $k_{1}(x)=\beta_{5} \tanh \eta_{p} x$. Substituting the above form of $k_{1}(x)$ in the secularity conditions and on evaluating the integrals and after rescaling $T \rightarrow \frac{-4}{3} T, \xi_{p} \rightarrow-4 \xi_{p}$ we obtain

$$
\eta_{p T}=\beta_{5} \eta_{p}^{2} \xi_{p}, \quad \xi_{p T}=-\beta_{5} \eta_{p}\left(\frac{3}{8} \eta_{p}^{2}+2 \xi_{p}^{2}\right)
$$

The above equations can be combined together to give $\eta_{p T T}+\frac{3 \beta_{5}^{2}}{8} \eta_{p}^{5}=0$, which on integrating once becomes $\left(\eta_{p T}\right)^{2}-\frac{\beta_{5}^{2}}{8} \eta_{p}^{6}=C_{3}$, where $C_{3}$ is an arbitrary constant of integration. In order to understand the effect of the present inhomogeneity on the velocity and amplitude of the soliton more transparently, we choose $C_{3}= \pm \frac{\beta_{5}^{2}}{8}$ for convenience. For instance when $C_{3}=-\frac{\beta_{5}^{2}}{8}$, the solution after integrating once can be written in terms of the complete elliptic integral of first kind, $F(\hat{\psi}, \hat{k})=3^{\frac{1}{4}}\left[2 C_{4}-\frac{\beta_{5}^{2}}{4} T\right]$, where $\hat{\psi}=\cos ^{-1}\left[\frac{1+(1-\sqrt{3}) \eta_{p}^{2}}{1+(1+\sqrt{3})} \eta_{p}^{2}\right]$ and the period is given by $\hat{k}=\frac{\sqrt{(2+\sqrt{3})}}{2}$. When $C_{3}=\frac{\beta_{5}^{2}}{8}$, using the transformation $\eta_{p}^{2}=\frac{1}{1+z^{2}}$, the equation after integrating once can be written as $\int_{y}^{\infty} \frac{d z}{\sqrt{z^{4}+3 z^{2}+3}}=\frac{-\beta_{5}^{2}}{8} T+C_{5}$, where $C_{5}$ is another constant of integration. The above equation can be integrated and $\mathrm{z}$ can be expressed in terms of Jacobian elliptic functions. Thus the final form of the amplitude $\eta_{p}$ and velocity $\xi_{p}$ read as $\eta_{p}=\frac{1}{\sqrt{(1+\sqrt{3})}}\left[\frac{1-\chi c n T}{1-\frac{1-\sqrt{3}}{1+\sqrt{3}} c n T}\right]^{\frac{1}{2}}, \xi_{p}=$ $\frac{3^{\frac{1}{8}}(1+\sqrt{3})}{\sqrt{16[(1+\sqrt{3})-(1-\sqrt{3}) c n T]}}\left[\frac{(1-\sqrt{3})}{(1+\sqrt{3})}\{1-\chi c n T\}^{\frac{-1}{2}}+\chi\left[1-\frac{(1-\sqrt{3})}{(1+\sqrt{3})} c n T\right]\{1-\chi c n T\}^{\frac{-3}{2}}\right] \operatorname{snT} T d n T$, where $\chi=\left[1-2 \sqrt{3}(1+\sqrt{3}) \eta_{p 0}^{2}\right]$, sn, cn and dn are Jacobian elliptic functions with the period as $\frac{1}{2} \sqrt{(2-\sqrt{3})}$ and $\eta_{p 0}$ is the initial amplitude of the soliton in this case. In Figs. 3a and 3b, we have plotted the velocity and amplitude of the soliton by choosing the initial amplitude of the 
soliton as $\eta_{p 0}=\frac{1}{2 \sqrt{3}} \frac{1-\sqrt{3}}{(1+\sqrt{3}) 2}$ for convenience. Unlike the case of quadratic inhomogeneity, in this case, due to the high nonlinear nature of the inhomogeneity the soliton flip and hence the magnetization reversal is not occuring very dominantly. The amplitude of the soliton infact oscillates doubly periodically smoothly with a marginal reversal in the amplitude. However, the velocity of the soliton shows dramatic turns at the points when it reverses or switches. It is observed that when the soliton amplitude changes from positive(negative) to negative(positive), it suddenly moves either forward or backward and on all other occasions it moves very slowly and the soliton is almost at rest. Thus, in this case, due to the high nonlinear nature of inhomogeneity the soliton is almost arrested and jumps suddenly forward or backward when the amplitude of the soliton reverses.

\section{Perturbed spin soliton}

Having analysed the nature of evolution of the amplitude and velocity of the soliton, now we construct the perturbed soliton by solving Eq.(5) after using the velocity and amplitude evolution equations corresponding to quadratic and tangent hyperbolic inhomogeneities. The perturbed solutions $\phi_{1}$ and $\psi_{1}$ can be derived using the particular solutions available for the homogeneous parts of the equations (5) namely $\left(\phi_{11}, \phi_{12}\right)$ and $\left(\psi_{11}, \psi_{12}\right)$ respectively. The particular solutions are given by $\phi_{11}=\operatorname{sech} \eta\left(\theta-\theta_{0}\right) \tanh \eta\left(\theta-\theta_{0}\right), \phi_{12}=-\frac{1}{\eta}\left[\operatorname{sech} \eta\left(\theta-\theta_{0}\right)-\frac{3}{2} \eta\left(\theta-\theta_{0}\right) \operatorname{sech} \eta\left(\theta-\theta_{0}\right) \tanh \eta(\theta-\right.$ $\left.\theta_{0}\right)-\frac{1}{2} \tanh \eta\left(\theta-\theta_{0}\right) \sinh \eta\left(\theta-\theta_{0}\right)$ and $\psi_{11}=\operatorname{sech} \eta\left(\theta-\theta_{0}\right), \psi_{12}=\frac{1}{2 \eta}\left[\eta\left(\theta-\theta_{0}\right) \operatorname{sech} \eta\left(\theta-\theta_{0}\right)+\sinh \eta(\theta-\right.$ $\left.\theta_{0}\right)$ ]. Knowing two particular solutions corresponding to the homogeneous part of the equations, the general solution can be written in the form $\phi_{1}=\delta_{1} \phi_{11}+\delta_{2} \phi_{12}-\phi_{11} \int_{-\infty}^{\theta} \phi_{12} F_{1} d \theta^{\prime}+\phi_{12} \int_{-\infty}^{\theta} \phi_{11} F_{1} d \theta^{\prime}$ and $\psi_{1}=\delta_{3} \phi_{11}+\delta_{4} \phi_{12}-\phi_{11} \int_{-\infty}^{\theta} \psi_{12} F_{2} d \theta^{\prime}+\psi_{12} \int_{-\infty}^{\theta} \psi_{11} F_{2} d \theta^{\prime}$ where $\delta_{1}, \delta_{2}, \delta_{3}$ and $\delta_{4}$ are constants. We construct $\phi_{1}$ and $\psi_{1}$ and hence $\hat{q}_{1}$ by evaluating the above integrals for the quadratic and tangent hyperbolic inhomogneities. The resultant solutions contain secular terms which make the solutions unbounded. We remove these secular terms by choosing the arbitrary constants in the solutions appropriately. Also the boundary conditions determine the more arbitrary constants available in the solutions. Construction of the solutions involve evaluation of several complicated integrals and very lengthy calculations. Therefore we desist from presenting the details of calculations here but give only the final form of the solutions. In the case of quadratic inhomogeneity the perturbed solution $\hat{q}_{1 s}$ is written as $\hat{q}_{1 s}=\left[\frac{3}{4} \Gamma\left(\Theta-\frac{3}{\eta_{s}}\right)+\beta_{0} \Delta \Theta+\frac{15 \beta_{2}}{2 \eta_{s}^{2}}-\frac{1}{2} \Delta\left[5 \beta_{2} \Theta^{2}-\beta_{1} \Theta+\frac{9}{2 \eta_{s}} \beta_{0}-\frac{1}{\eta_{s}} \hat{\beta} \Theta\right]+\right.$ $\left.\left(\frac{5 \beta_{2}}{\eta_{s}^{2}}+\frac{\beta_{0} \Delta}{\eta_{s}^{2}}\right) \ln \cosh \eta_{s} \Theta\right] \operatorname{sech} \eta_{s} \Theta+\left[\frac{-15 \beta_{2}}{2 \eta_{s}^{2}}+\frac{7}{4 \eta_{s}} \Gamma+\frac{1}{4} \Delta\left[15 \beta_{2} \Theta^{2}+7 \beta_{0}\right]-\frac{4 \beta_{2}}{\eta_{s}^{2}} \ln \cosh \eta_{s} \Theta\right] \operatorname{sech}^{3} \eta_{s} \Theta+$ $\left[\frac{1}{2} \Delta \Theta\left(5 \beta_{2} \eta_{s}^{2} \Theta-\beta_{1} \eta_{s}^{2}+\frac{11 D}{2} \eta_{s}-\frac{6 \beta_{2}}{\eta_{s}}-\frac{3 \beta_{2}}{\eta_{s}} \ln \cosh \eta_{s} \Theta\right)\right] \operatorname{sech} \eta_{s} \Theta \tanh \eta_{s} \Theta+i\left[\left[D+\left[-\frac{1}{2} D+\wp \Theta+\right.\right.\right.$ $\left.\left.\frac{1}{\eta_{s}}\left[\xi_{s} \eta_{s}^{2} \Theta^{2}+\frac{\beta_{1}}{2} \Theta+D-3 \eta_{s}\right]\right] \frac{\Theta}{2}\right] \operatorname{sech} \eta_{s} \Theta+\left[\left[-\wp \Theta-\xi_{s} \eta_{s}\left[\beta_{2} \Theta^{2}-\beta_{1} \Theta+\beta_{0}\right] \frac{\Theta}{2}\right]-2 D \eta_{s}+D-\right.$ $\left.\left.\frac{\xi_{s} \beta_{2}}{\eta_{s}^{2}}\right] \operatorname{sech}^{3} \eta_{s} \Theta+\left[\frac{1}{2}\left[\frac{1}{\eta_{s}} \wp \Theta-2 D-\frac{11}{2} \xi_{s} \beta_{2} \Theta^{2}+\xi_{s} \beta_{0}\right]\right] \operatorname{sech} \eta_{s} \Theta \tanh \eta_{s} \Theta+\left[\frac{1}{\eta_{s}} \beta_{2} \xi_{s} \Theta\right] \operatorname{sech}_{s} \Theta \ln \cosh \eta_{s} \Theta\right]$ where $\Gamma=\left(\xi_{s} \theta_{0 T}+\sigma_{0 T}+2 \beta_{2}\right), \wp=\hat{\beta} \xi_{s}\left(\eta_{s}-2\right) \Theta=\left(\theta-\theta_{0}\right), \Delta=\left(\eta_{s}^{2}-\xi_{s}^{2}\right)$ and $D=\beta_{0} \xi_{s} \eta_{s}$. Similarly 
$\hat{q}_{1 p}$ in the case of hyperbolic inhomogeneity is also constructed.

\section{Conclusions}

In this paper, we studied the effect of nonlinear inhomogeneity in bilinear and biquadratic exchange interactions on the spin soliton of a classical continuum Heisenberg ferromagnetic spin chain, the dynamics of which is governed by an inhomogeneous generalized higher order NLS equation upon mapping the spin chain onto a moving space curve. The effect of inhomogeneity was understood by carrying out mulitple scale perturbation analysis on an inhomogeneous NLS equation and by analysing the evolution of the velocity and amplitude of the soliton. As examples, we considered quadratic and tangent hyperbolic-type inhomogeneities. The results of the perturbation analysis shows an interesting phenomenon of soliton flipping leading to magnetization(spin) reversal and in the ferromagnetic medium. When the soliton moves along the spin chain with quadratic-type inhomogeneity, starting from rest with a finite amplitude, the soliton amplitude grows up and is accelerated and when the velocity reaches a maximum value, it suddenly flips(magnetization reversal), then slows down, once again flips and this phenomenon is found to occur doubly periodically for an indefinite time. However, when the inhomogeneity is in the form of a tangent hyperbolic function, the soliton jumps back and forth and the amplitude in this case changes smoothly, and doubly periodically however only with a marginal amount of negative amplitude. Finally, we also constructed perturbed solitons in both the cases of inhomogeneity. The above spin soliton flipping

phenomenon which leads to magnetization reversal in ferromangetic medium is expected to have potential applications in magnetic memories and recording.

\section{Acknowledgments}

The work of M.D forms part of a major research project sponsored by the

DST, Govt. of India. The major part of the work is carried out at the Abdus Salam ICTP, Trieste, Italy under the Regular Associateship of M.D. The authors acknowledge the support from the Abdus Salam ICTP, Trieste, Italy.

\section{References}

1 R. Kikuchi, J. Appl. Phys. 27, 1352 (1957).

2 A. Hubert, and R. Schäfer, Magnetic Domains (Springer-Verlag, Berlin, 1998).

3 Y. Iwasaki, J. Magn. Magn. Mater. 240, 395 (2002). 
4 R. W. Chantrell, M. Wongsam, J. D. Hannay, and O. Chubykalo, Comp. Mater. Sci. 17, 483 (2002).

5 M. Bauer, R. Lopusnik, J. Farsbender, and B. Hillebrands, J. Magn. Mag. Mater. 218, 165 (2000).

6 M. Bauer, J. Fassbender, B. Hillebrands, and R. L. Stamps, Phys. Rev. B61, 3410 (2000).

7 L. Landau, and E. Lifshitz, Phys. Z. Sowjetunion 8, 153 (1935).

8 M. Lakshmanan, Phys. Lett. A61, 53 (1977).

9 K. Porsezian, M. Daniel, and M. Lakshmanan, J. Math. Phys. 33, 1807 (1992).

10 M. Daniel, K. Porsezian, and M. Lakshmanan, J. Math. Phys. 35, 6498 (1994).

11 L. Kavitha, and M. Daniel, Phys. Rev. E (Submitted).

12 J. J. Stoker, Differential Geometry (Wiley, New York, 1969).

13 J. Weiss, M. Tabor, and G. Carnevale, J. Math. Phys. 24, 522 (1983).

14 M. Lakshmanan, and R .K. Bullough, Phys. Lett. A80, 287 (1980).

15 M. Lakshmanan, and R. Ganesan, Physica A132, 117 (1985).

16 K. Porsezian, M. Daniel, and R. Bharathikannan, Phys. Lett. A156, 206 (1991).

17 Y. Kodama, and M. J. Ablowitz, Stud. Appl. Math. 64, 225 (1981).

18 P. F. Byrd, and M. D. Friedman, Handbook of Elliptic Integrals for Engineers and Scientists (Springer-Verlag, Berlin, 1971). 